Full Length Article

\title{
Screening for modulators of autism spectrum disorder using induced human neurons
}

\author{
Sumitha Rajendra Rao ${ }^{\mathrm{a}}$, Ana Kostic ${ }^{\mathrm{b}}$, Pierre Baillargeon ${ }^{\mathrm{a}}$, Virneliz Fernandez-Vega ${ }^{\mathrm{a}}$, \\ Mitzy Rios de Anda ${ }^{\mathrm{b}}$, Kelty Fletcher ${ }^{\mathrm{a}}$, Justin Shumate ${ }^{\mathrm{a}}$, Louis Scampavia ${ }^{\mathrm{a}}$, Joseph D Buxbaum ${ }^{\mathrm{b}}$, \\ Timothy P. Spicer ${ }^{\text {a,* }}$
}

${ }^{a}$ The Scripps Research Molecular Screening Center, Department of Molecular Medicine, Scripps Research, Jupiter, FL 33458, USA

${ }^{\mathrm{b}}$ Seaver Autism Center for Research and Treatment, Department of Psychiatry, Icahn School of Medicine at Mount Sinai, New York 10029, USA

\section{A R T I C L E I N F O}

\section{Keywords:}

Autism spectrum disorder

Neuroprotection

High throughput screening

Induced neurons

Small molecules

\begin{abstract}
A B S T R A C T
Autism Spectrum Disorder (ASD) is a heterogeneous neurodevelopmental disorder. There are no drugs to treat the core symptoms. De novo mutations often play an important role in ASD and multiple high-risk loci have been identified in the last decade. These mutations range from copy number variants to small insertion/deletion and single nucleotide variants. Large-scale exome sequencing has identified over 100 risk genes that are associated with ASD. Both etiological heterogeneity and unavailability of human neurons remain major hurdles in understanding the pathophysiology of ASD and testing of new drug candidates. Hence, the most achievable and relevant model to screen for potential drugs is human neurons from inducible pluripotent stem cells (iPSCs), including those from individuals with genetic mutations. In this study, we tested stem cells from individuals carrying mutations in ADNP, FOXP1 or SHANK3. They were scaled and reprogrammed to glutamatergic neurons and assessed for the effects of their specific mutations on neurite outgrowth. High Content Analysis allowed us to observe phenotypic differences between ASD neurons compared to controls, in terms of neuron number, neurite number and neurite length per neuron. Further, neurons were derived from both patient derived and genetically modified iPSCs with DDX3X mutation which were tested against 5088 drug like compounds. We assessed individual compound effects on the induced neurons to determine if they elicited changes that would indicate neurite growth (neuroprotection) or, alternatively, reduce outgrowth and hence appear neurotoxic. This report includes all methods, phenotypic outcomes, and results for the largest ASD small molecule screening effort done to date.
\end{abstract}

\section{Introduction}

Autism spectrum disorder (ASD) is a serious neurodevelopmental disorder characterized by impaired social and intellectual communications and restrictive and/or repetitive behaviors. Estimated ASD prevalence rate is 16.8 per 1,000 in children, with males affected four times more often than females. ASD is diagnosed by behavioral changes that are evident as early as 18 months and become stable by the age of 3 [1]. Currently, ASD is diagnosed by the Diagnostic and Statistical Manual of Mental Disorders criteria and presents a major global health concern with no drugs approved for core symptoms and high burden on patients and their families as well as on the healthcare system. Genetic, epigenetic, and environmental factors are posited to contribute to ASD, but the specific causes are just now being elucidated. Both early twin and recent national epidemiological risk studies have shown very high heritability, with both common and rare genetic variation contribut- ing to risk ASD [2-5]. Tremendous progress has been attained in highthroughput sequencing to identify genes that, when mutated, confer high risk for ASD: most recently, exome sequencing data from 35,584 samples identified 102 high-confidence ASD risk genes [6].

Non-genetic and genetically modified rodent models showing ASDlike behavioral phenotypes have been instrumental in understanding the pathobiology of ASD $[7,8]$. However, species differences including development of neocortex that dictates higher order functions differentiates animals and humans, and impacts the usefulness of these models in recapitulating ASD [9]. This is complicated by the fact that ASD is a neurodevelopmental disorder and information gained from postmortem brain tissue is minimal. In the recent past, use of human stem cells such as embryonic stem cells (ESCs) and human induced pluripotent stem cells (hiPSCs) derived from transcriptional re-programming of somatic cells have proven to be extremely useful in disease modeling, toxicity testing and regenerative medicine $[10,11]$. Stem cells can be maintained

\footnotetext{
* Corresponding author at: Scripps Research-Florida, 130 Scripps Way, Jupiter, FL 33458, USA.

E-mail address: spicert@scripps.edu (T.P. Spicer).
} 
in culture for many passages, similar to immortalized cell lines, and they can be differentiated into a myriad of cell types. Fortunately, iPSCs can now be used to generate large, homogenous populations of various neural cell types, and recently have proven to be scalable to test intrinsic cell responses to large-scale pharmacological interventions $[10,11]$. When considering complex disorders like ASD, it is also possible that the impact of driver mutations could be modified by differences in the genetic background of individuals. Clustered regularly interspaced short palindromic repeat (CRISPR) with CRISPR-associated protein-9 nuclease (Cas9) (CRISPR/Cas9) gene editing system can circumvent this issue by generating disease specific mutations in hiPSCs that can mimic disease phenotype as virtual patients. With this advancement multiple genes can be perturbed, even simultaneously, and studied in parallel and compared to their isogenic control cell lines that do not carry the mutation.

Former studies used ESCs/ hiPSCs carrying mutations in GPD2 and SHANK3 genes which were differentiated into cortical excitatory or inhibitory neurons and olfactory placodal neurons to study ASD pathogenesis [12-15]. In one of the studies, cortical neurons were derived using small molecules and were screened against 202 compounds to identify SHANK3 regulators [16]. While this set the stage for small molecule screening of ASD, the published protocols were very lengthy and lacked the robustness required for High Throughput Screening (HTS). Thus, there was a dire need for developing humanized in vitro models of ASD amenable for HTS. In an earlier study [10], we have shown that freezer ready glutamatergic neurons can be generated from commercially available stable human episomal Cas9 transformed iPSC cell line based on Ngn2 transcription system [10,17]. Neurite outgrowth was used as an endpoint/phenotypic readout and this method was found to be robust and compatible with HTS. Taking this idea forward, here we have adopted the established robust neuron scaling protocol and combined it with CRISPR/Cas9 technology to develop high throughput ASD relevant disease models. hiPSCs were edited using CRISPR/Cas9 to introduce mutations in the ADNP, FOXP1, DDX3X or SHANK3 genes and were reprogrammed transcriptionally to induced glutamatergic neurons (iNs). Comparison of neurite outgrowth using High Content Assay (HCA) revealed phenotypic differences with the ASD variants, compared to isogenic control. This prompted us to investigate mutant iNs derived directly from an ASD patient and a sibling control. Herein we describe how these patient/ CRISPRed hiPSCs derived neurons were screened against Maybridge library to identify neuroprotective molecules that could be used for treating ASD.

\section{Materials and methods}

All reagents used were purchased from Thermo Fisher Scientific unless and otherwise mentioned. Prior to use, reagents were warmed up to $15-25^{\circ} \mathrm{C}$ at room temperature and not in a water bath at $37^{\circ} \mathrm{C}$.

\section{CRISPR/Cas9 genome editing of hiPSC}

The following CRISPR/Cas9-engineered hiPSC lines were used in this study: i) The male parental line ASE-9203 (the control for lines ii-iv); ii) the FOXP1 p.Arg525* mutation (line C1577); iii) a heterozygous SHANK3 100kb deletion (including SHANK3, ACR, RABL2B genes) (line C1455, clone XXX); iv) a heterozygous SHANK3 100kb deletion (including SHANK3, ACR, RABL2B genes) (C1455, Clone 10), v) the ADNP p.Asn832Lysfs* mutation (C1581), vi) the female parental line NSB3182 (the control for line vii); and, vii) the DDX3X pHis512Argfs*5 mutation (C1630). Generation of engineered cell lines was performed by Applied Stem Cell (Carlsbad, CA) using all mutations introduced by CRISPR/Cas9 as described previously [18].

\section{Derivation of hiPSC}

The following additional hiPSC lines were used in this study: i) CC1368.202, clone 3 from an individual with the most common recurrent ADNP pTyr719* mutation; ii) CC1368.202, clone 4 from the same individual; and iii) CC1368.201, a sibling derived control for the individual carrying the ADNP p719* mutation. These cell lines were generated at the Black Stem Cell Institute Core Facility, Icahn School of Medicine at Mount Sinai, New York, as previously described [19]; after reaching passage 10, hiPSC colonies were transitioned to feeder-free conditions using Matrigel-coated plates (Corning) and mTeSR1 medium (Stem Cell Technology).

\section{hiPSC revival, propagation, and scaling}

Six well tissue culture plates (cat no. 140675) were prepared for growing hiPSCs by coating with Vitronectin (cat no. A14700) 1:100 in 1X DPBS (cat no. 14190094) and incubated at room temperature (RT) for $1 \mathrm{hr}$. Control, CRISPRed and patient derived hiPSCs were revived from liquid nitrogen and thawed in a water bath at $37^{\circ} \mathrm{C}$. When the last ice crystal was seen, cells were transferred to a centrifuge tube with $5 \mathrm{~mL}$ of prewarmed stem cell complete medium composed of Stem Flex Medium (cat no. A3349401) with 1\% Antibiotic-Antimycotic (cat no. 15240062) and centrifuged at $1000 \mathrm{rpm}$ for 5 minutes. Vitronectin was removed from the tissue culture plates prior to addition of hiPSCs. The cell pellet was re-suspended in stem cell complete medium supplemented with RevitaCell (1:100) (cat no. A2644501) and seeded on to vitronectin coated plates. hiPSCs were incubated at $37^{\circ} \mathrm{C}$ with $5 \% \mathrm{CO}_{2}$ and $95 \%$ Relative humidity (RH) and routinely observed for colony formation. Each of the hiPSC cell lines were confirmed to be mycoplasma negative by a third-party vendor (cat. no. M-250, Bionique Laboratories, Saranac Lake, NY). hiPSCs were passaged at $70-80 \%$ confluency either with ReLeSR ${ }^{\mathrm{TM}}$ (cat no. 05872, Stem Cell Technologies,) or TrypLE ${ }^{\mathrm{TM}}$ Select Enzyme (cat no. A1217703). hiPSC colonies were washed with $1 \mathrm{X}$ DPBS and exposed to a thin film of ReLeSR ${ }^{\mathrm{TM}}$ for a minute at RT. ReLeSR was aspirated and incubated at $37^{\circ} \mathrm{C}$ for $3-5$ minutes until the colonies were dissociated. The flask was tapped on the sides to detach cell aggregates, fresh stem cell complete medium was added and distributed into pre-coated T75 tissue culture flasks (BD Cat no. 353136) at a ratio of 1:2. Likewise, hiPSC were scaled to T175 flasks (Cat no. 159910) for maintenance. During single cell passaging, hiPSC colonies were washed with 1X DPBS, and incubated with $1 \mathrm{X}$ TrypLE Select at $37^{\circ} \mathrm{C}$ for 5 minutes. Once the colonies were detached, an equal volume of complete medium was added to stop trypsinization, cell number counted and centrifuged at $1000 \mathrm{rpm}$ for 5 minutes. The cell pellet was resuspended in fresh medium and seeded at a density of $2 \times 10^{6}$ cells per T75 flasks (cat no. 13-680-65, Fisher Scientific).

Alternately, hiPSCs dissociated using TrypLE select were cryopreserved as 5 million cells in $1 \mathrm{~mL}$ of ice-cold PSC cryopreservation medium (cat no. A2644601) per cryovial, stored in a Nalgene's Mr. Frosty Cryo $1{ }^{\circ} \mathrm{C}$ Freezing Container (cat. no. 5100-0001) at $-80^{\circ} \mathrm{C}$ overnight, barcodes were added, logged, and transferred to liquid nitrogen for long term storage.

\section{Immunostaining for pluripotency}

hiPSCs were dissociated with ReLeSR ${ }^{\mathrm{TM}}$ and plated onto Vitronectin coated 384 well plates (cat no. 789068-T Greiner bio-one) and were incubated at $37^{\circ} \mathrm{C}$ with $5 \% \mathrm{CO}_{2}$ and $95 \% \mathrm{RH}$. The next day, cells were fixed with $4 \%$ paraformaldehyde (cat no. 15710, Electron Microscopy Sciences) in 1X DPBS at RT for 20 minutes. Cells were washed in $1 \mathrm{X}$ DPBS (cat no. 14190094) and incubated with 0.1M Glycine for 20 minutes at RT. Cells were permeabilized with $0.3 \%$ Triton- X 100 in $1 \mathrm{X}$ DPBS for 10 minutes followed by blocking with 3\% Bovine Serum Albumin (BSA) for 45 minutes at RT. The cells were stained with OCT4 polyclonal antibody (cat no. PA5-27438) or SSEA4 monoclonal antibody 
(cat no. MC-813-70) in 1X DPBS with $1 \%$ BSA and incubated at $+4^{\circ} \mathrm{C}$ overnight. Next day, cells were washed and labelled with secondary antibodies - Alexa flour 488 or 647 (1:500) (cat no. A11008 or A21235) for $2 \mathrm{hrs}$ at RT, counter stained with Hoechst and washed twice with 1X DPBS. Fluorescent images were acquired at $5 \mathrm{x}$ objective in a CellInsight imaging reader (Thermo Fisher, Waltham, MA) with appropriate filters.

\section{Reprogramming hiPSCs into glutamatergic neurons}

hiPSCs were reprogrammed to glutamatergic neurons by following previously established protocol with slight modifications [20]. Lentiviral constructs with plasmid vectors TetO-NGN2- Puro (Addgene Accession ID: 52047) and FUW- MrTA (Addgene Accession ID: 20342) were purchased from VectorBuilder Inc. USA. hiPSCs were split with TrypLE select or ReLeSR and were seeded on Vitronectin coated tissue culture flasks at a density $2 \times 10^{6}$ cells / T75 flasks (Parent, FOXP1 and SHANK3 clones) or $3 \times 10^{6}$ cells / T175 flasks (ADNP patient derived clones and sibling) or $5 \times 10^{6}$ cells / T175 flasks (DDX3X mutant and isogenic control) (Day 1). On Day 4 - Parent, FOXP1 and SHANK3 clones were infected with lentiviral constructs at 1 Multiplicity of Infection in stem cell complete medium supplemented with 1X RevitaCell. Similarly, on Day 2 , patient derived and DDX3X hiPSCs were infected. The next day, media was replaced with Neurobasal medium (cat. no. 21103049) containing Glutamax ${ }^{\mathrm{TM}}$ Supplement (cat. no. 35050061), B-27 ${ }^{\mathrm{TM}}$ Supplement (cat. no. 17504044), 1\% Antibiotic- Antimycotic and $2 \mu \mathrm{g} / \mathrm{mL}$ doxycycline (cat. no. 10592-13-9, Frontier Scientific). After 24 hours, the reprogrammed cells were refreshed with the same medium and selected by addition of $2 \mu \mathrm{g} / \mathrm{mL}$ of Puromycin for 2 more days. The induced Neurons (iNs) were then dissociated with Accutase (cat. no. A1110501) and either plated on PDL coated plates or cryopreserved in Synth A freeze (cat no. 1254201) and stored in liquid nitrogen following the steps used for hiPSCs. The timelines used for neural induction of different hiPSCs are presented in Fig. 2A, Sup. Fig. 2A and Sup. Fig. 6A.

\section{Optimizing neurite outgrowth staining and neuro-profiling algorithm}

iNs were plated on to black clear bottom Poly-D-Lysine coated, square IQ-EB 384 well plate (cat. no. ABE2-41201-B, Aurora Biotechnologies, Whitefish, MT) at a density of 2500 cells in $30 \mu \mathrm{L} /$ well using a manual multichannel pipettor, or as 1300 cells in $5 \mu \mathrm{L} /$ well on to 1536 well plate (cat no. EBA1-41000B-PDL Aurora Biotechnologies, Whitefish, MT) using a Flying Reagent Dispenser (FRD, Aurora Biosciences) and incubated at $37^{\circ} \mathrm{C}$ with $5 \% \mathrm{CO}_{2}$ and $95 \% \mathrm{RH}$. Plating medium was composed of Neurobasal medium, $1 \mathrm{X}$ Glutamax ${ }^{\mathrm{TM}}$ Supplement, 1X B$27^{\mathrm{TM}}$ Supplement, $1 \%$ Antibiotic- Antimycotic and neurotrophic factors such as BDNF, GDNF and NT3 (20ng/mL) (Peprotech, Rocky Hill, NJ). After overnight incubation, the differentiated neurons were treated with $100 \mathrm{~nL}$ and $30 \mathrm{~nL}$ control compounds for 384 and 1536 well formats respectively or DMSO using a pintool transfer unit (Kalypsys/ GNF). Control compounds included Brefeldin A, Blebbistatin, Rho Kinase Inhibitor$\mathrm{Y}-27632$ and Rotenone at a final concentration of $10 \mu \mathrm{M}$ with two-fold dilutions, for 10 or 20 points, which were used for estimating concentration response. After $48 \mathrm{hrs}$ of incubation at $37^{\circ} \mathrm{C}$, iNs were fixed with $4 \%$ paraformaldehyde and stained with Neurite Outgrowth Kit (cat no. A15001) following manufacturer's instructions and counterstained with Hoechst (1:1000) (cat no. H3570). Images were acquired at 5x objective in the CellInsight. Initially, seeding of iNs in 384 well plates and washing steps during staining were performed manually. This led to variations within different wells of the same samples and subsequently heightened standard deviations. Further these steps were optimized by using the FRD for seeding iNs and a Bluewasher (Blue Cat Bio $\mathrm{GmbH}$ ) with gentle spin mode for washing steps.

The neuronal profiling algorithm optimized by Sridharan et al., $2019^{10}$ was followed. Briefly, nuclear staining in channel 1 (Ch1) (386 $\mathrm{nm}$ ) was used for primary object identification and cell bodies in channel 2 (Ch2)/ neurites stained by the neurite outgrowth kit (546 nm) were used for neuron count and neurite identification. The output parameters were exported using Z' ranking tool and the following parameters of the 'well features' were considered for analysis: i) valid nucleus count - total number of nuclei identified in the field (Ch1 object selection), ii) neurite total count- total number per field of all neurites selected for analysis in the well, and iii) neurite total length- total neurite length per field of the neurites selected for analysis in the well (Sup. Fig. 3).

\section{Data normalization}

An HCA neuronal profiling algorithm that was available on the CellInsight software was implemented, and it worked well. When output parameters of the 'well features' were checked, number of nuclei did not correspond to the number of neurons indicating the data not being normalized to real number of neurons in that field. Henceforth, 'cell features' for output parameters was used to capture the phenotypic differences based on neuron per se where cell bodies (neurons) in Ch2 were used for analysis. Thus, per neuron is the total value of a feature/output parameter divided by total number of neurons. Data were re-analyzed with new parameters viz ., i) valid neuron count - number of valid neurons identified in the field based on staining in Ch2, ii) neurite total count per neuron and iii) neurite total length per neuron. Thus, we captured the phenotypic differences based on neuron number per se (Fig. 1C, i to iii). Compared to control iNs treated with vehicle only, all mutant variants showed significant reduction of neurite number per neuron (Fig. 1C, iii). In fact, after multiple rounds of optimization for these 3 parameters, we settled collectively on assessing the outcomes of the HCA and screening on neurite count per neuron as it appeared to give the most significant result which would ultimately aid in identifying neuroprotective small molecules.

\section{Maybridge HitFinder library screening and data analysis}

As part of the Scripps Drug Discovery Library of $>666 \mathrm{~K}$ molecules we have the Maybridge HitFinder library contains $14.5 \mathrm{~K}$ compounds that fit Lipinski guidelines for "Drug-likeness" (cLogP $\leq 5, \mathrm{H}$-bond acceptors $\leq 10$, H-bond donors $\leq 5$, Molecular Weight $\leq 500$ ) [21]. iNs were screened against 5088 of the Maybridge compounds, of which, 3626 compounds had a central nervous system multiparameter optimization (CNS MPO) desirability score $\geq 4$. Frozen iNs from ADNP sibling, ADNP mutant clone 4, DDX3X isogenic control and DDX3X mutant were used for screening. The final HCS protocol is summarized in Sup.Table.1.

In terms of controls, we used $\mathrm{N}$ of 24 control wells on all individual plates and looked for systemic effects. Neurons are fragile cells and could get easily lifted/washed off during formaldehyde fixation and staining procedures causing higher standard deviations and the Z's were not passing. To compensate for the variations, we tested all 5088 compounds in triplicates and average response of the activity (neurite total count per neuron) was used for identifying hits. Data was uploaded in the Scripps institutional database and average $\%$ response activity was calculated using the following formula.

$\%$ Activity $=100 \times \frac{\text { Testwell }- \text { MedianLow Controls }}{\text { MedianHighControls }- \text { MedianLowControl }}$

Hits with neurotoxic activity were identified using rotenone as high control. Data binning and histogram analysis were performed, and a hit cut off mean $+3 S D$ was used to select the hits for neurotoxicity. Since there is no known neuroprotective compound and iNs responded weakly to Blebbistatin, we used the HCA raw output values for hit identification and a hit cut off mean +2 SD for neuroprotection. The controls used in this study were tested and published earlier [10].

\section{Cytotoxicity assay}

Cytotoxicity of the neurotoxic hits were assessed on HEK-293 cells using an earlier established protocol [22]. HEK 293 cells were used as 
A

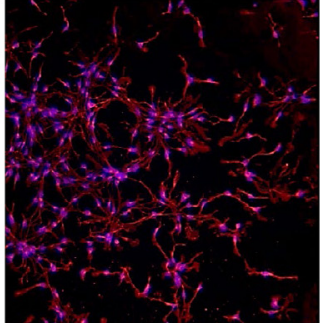

Parent

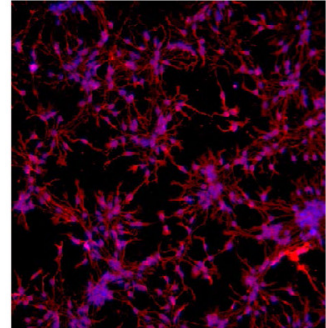

ADNP

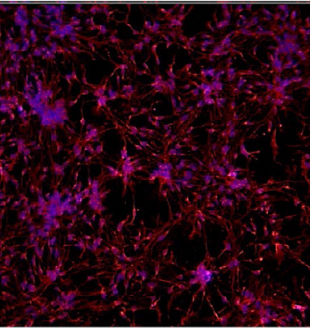

FOXP1

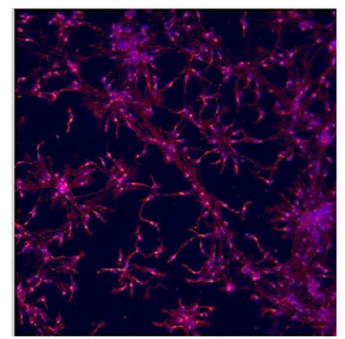

Shank3 Clone10



Shank3 Clone1

B

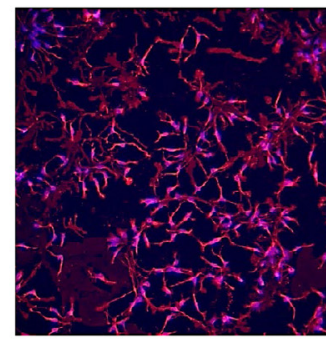

Image - Acquired

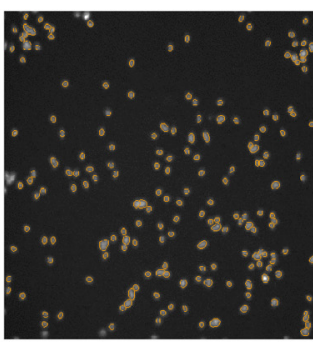

Channel 1 (Ch1)- Nucleus

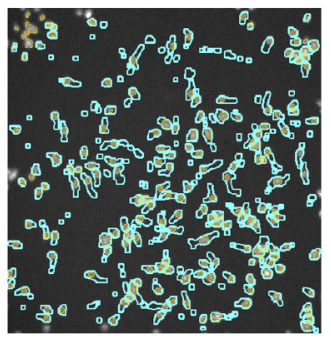

Channel 2 (Ch2)- Cell bodies

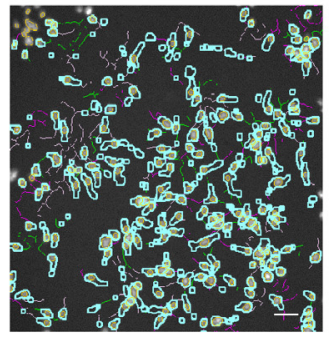

Channel 2- Neurites
C



ii

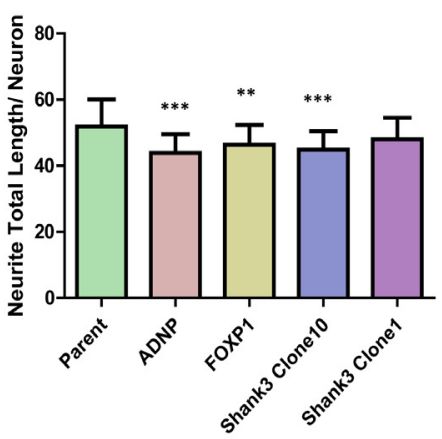

V

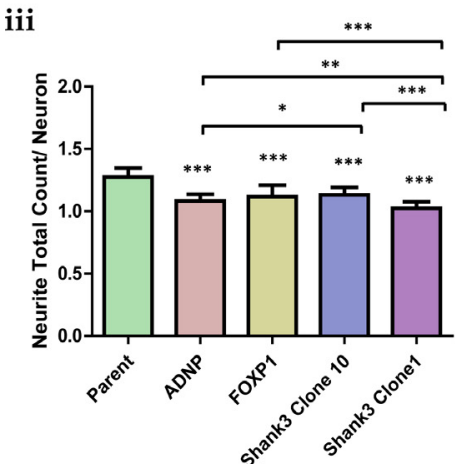

iv

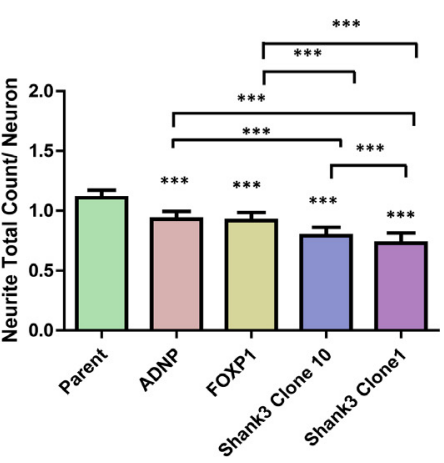

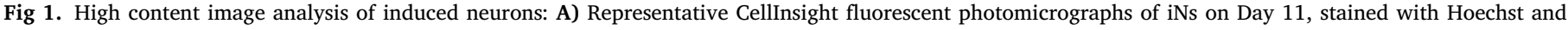

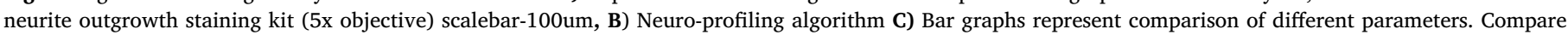



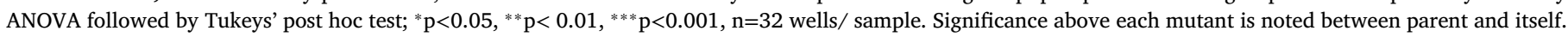
Bracketed significance is noted between clones. 
they are easier to scale and expedite the assay compared to iNs. This was done as it is far more cost and time effective considering the reagents required for scaling hiPSCs and inducing them to neurons. Frozen ready to use HEK cells were thawed and 500 cells in a $5 \mu \mathrm{L}$ of Dulbecco's Modified Eagle's Media (DMEM) containing $10 \% \mathrm{v} / \mathrm{v}$ fetal bovine serum and $1 \mathrm{X}$ antibiotic mix (penicillin and streptomycin) were plated into each well of 1536-well tissue culture-treated microtiter plates. Immediately, test compounds were pinned using $30 \mathrm{~nL}$ pintool along with control compounds doxorubicin (150 $\mu \mathrm{M}$ final concentration) and rotenone and brefeldin A. After $48 \mathrm{hrs}$ of incubation, CellTiter-Glo (Promega Corp., Madison, Wisconsin) reagent was added, and luminescence measured for 5 seconds on the ViewLux imaging plate reader. The percent inhibition for each compound was calculated using DMSO as low control and doxorubicin as high control.

\section{Statistical analysis}

The differences between and within the experimental groups were analyzed using one-way ANOVA followed by Tukey's post hoc test. Data are represented as Mean $\pm \mathrm{SD}$, scatterplots and bar graphs were made using GraphPad Prism (GraphPad Software, La Jolla, CA). Dose response box plots were generated using RStudio version 1.3.1093 [23] with R version 4.0 [24]. Dose response box plots also utilized the ggplot2 library for R [25]. Resulting dose response box plots were automatically exported from RStudio in PNG image format for inclusion in a web-based report that provided both in-house researchers and external collaborators with convenient access to results across experiments.

\section{Results}

\section{Scaling of CRISPRed / patient derived hiPSCs}

Isogenic and CRISPRed mutant variants of hiPSCs were propagated in culture. They adhered, started forming colonies that were closely packed, with well-defined boundaries. These cells expressed OCT4 that co-localized with Hoechst in the nucleus, and the cell surface glycosphingolipid stage specific embryonic antigen-4 (SSEA-4), indicating pluripotency (Sup. Fig. 1). The colonies were passage and scaled in T75 flasks, followed by T175 flasks. The yield per T175 flask ranged from $1.3 \times 10^{7}$ cells to $3.2 \times 10^{7}$ cells per flask. More than 15 cryovials of hiPSCs were cryopreserved in liquid nitrogen with 5 million cells/ vial.

\section{Developing an in-vitro humanized model of ASD amenable to HTS}

We were successful in differentiating all the five CRISPRed hiPSCs under study into iNs which adhered and displayed neuronal processes that interacted with each other (Sup. Fig. 2). Neurons stained with neurite staining kit are shown in (Fig. 1A\&B). Significant differences were observed in the neurite total count and neurite total length between the parent and other mutant iNs (Fig. 1C ii and iii). ADNP is a neuroprotective protein, and its partial or complete knockdown leads to cognitive impairments or affects brain development in mice. ADNP is also involved in neuronal spine density and expression of certain synaptic proteins [26]. FOXP1 is associated with the formation and integrity of radial glial cells during corticogenesis [27]. Thus, mutation in the ADNP or FOXP1 in the hiPSCs might have resulted in the reduced neurite total count and neurite total length of the derived iNs. Placodal neurons with SHANK3 mutations had increased primary neurite formation with reduced neurite elimination [28] and this contrasts our observation with reduced neurite count / length of iNs with SHNAK3 mutation. Heterogeneity was observed between the two independent experiments performed.

\section{Validating iNs for screening and assay miniaturization}

With the intent to complete a large scale HTS against the Maybridge library, we required large batches of iNs which were cryo-banked af- ter 8 days of neural induction. Next, we sought to check the viability and reproducibility of frozen iNs by thawing, re-plating, and comparing with directly plated iNs. The number of valid neurons following freeze thaw was similar in the isogenic control, and they were reduced in the mutant variants (Fig. 1C, i vs iv). The other output parameters such as total neurite count and neurite total length output were normalized to the valid neuron count per well and the resulting data were significantly reduced in mutant variants compared to the isogenic control, which is similar to directly plated neurons (Fig. 1C, iii vs v). To validate iNs for HTS screening, they were exposed to neurotoxic or neuroprotective control compounds including Brefeldin A, Rotenone, Blebbistatin and a RhoKinase inhibitor and dose response determined. The response of iNs from all five cell lines was as expected, wherein neuroprotective compounds Blebbistatin and RhoKinase inhibitor increased neuron number, total neurite count and length per neuron, whereas the neurotoxic compounds Brefeldin A and Rotenone reduced those: particularly at higher concentrations. Brefeldin A was found to be highly toxic compared to Rotenone and data were consistent and less variable (Sup. Fig. $4 \& 5$ ). As a final step, the assay was miniaturized to 1536 well format. Data from 384 and 1536 formats were comparable.

\section{HTS screening with ASD patient derived iNs}

Having validated the screening approach across multiple independent mutations, we carried out the HTS. We used (i) iPSC lines derived from a patient with ADNP mutation and (ii) an isogenic DDX3X mutant for this part of the screening effort. The justification towards these cells vs. others was defined by availability of genotypically defined and scalable iPSCs from patient isolates that including a WT pair. In other words, the patient derived hiPSCs available and used herein are the closest resource we must recapitulate the real ASD pathology. Among the two different ADNP mutant clones tested, neurite total count varied substantially. ADNP mutant clones had reduced neurite total length per neuron compared to sibling iNs (Fig. 2). This is similar to that observed with the CRISPRed ADNP iNs. ADNP clone 4 was chosen for the further experiments as it had better growth and yield compared to clone 3 . Here, the observed basal phenotypic difference between the sibling and the mutant highlights the importance of how they may differ from each other as it relates to their ASD features which, warrants further interrogation in future toward determining the neuronal spine density and expression of synaptic protein under these conditions. ADNP and DDX3X iNs responded to control compounds as expected (Fig. 3, 4A \& B \& Sup. Fig. $6,7)$.

We carried out the HTS using the Maybridge library vs iNs derived from ADNP clone 4, its sibling control, DDX3X mutant, and its isogenic control. Hits were identified based on neurite total count per neuron, which we had previously determined to be the most robust and logical output and are listed in Sup. Table 2. Histogram and binning analysis of the neuroprotective and neurotoxic hits are presented in Fig. 4C\&E, Sup. Fig. 8 A\&C. Looking at the neuroprotective hits there was no overlap between the ADNP iNs (sibling and mutant clone 4) (Fig. 4D) and DDX3X iNs (isogenic control and mutant) (Sup. Fig. 8B) and there were no common neuroprotective hits between all the four iN types tested (Fig. 5A). When looking at negative modulators of neurite count per neuron, two common hits were identified between the ADNP sibling and mutant clone 4 (Fig. 4F), and 64 common neurotoxic hits were found between DDX3X isogenic control and mutant (Sup. Fig. 8D). Overlapping neurotoxic hits between all four iN types are represented in the Venn diagram as shown in Fig. 5B, C.

\section{Dose response experiments with HTS hits}

After the primary screen a histogram analysis of the distribution of the raw outcome of the increase in neurites per neuron was done as shown in Figure 4. A total of 147 neuroprotective hits (all the hits identified with the 4 different iNs tested) were selected for dose response 


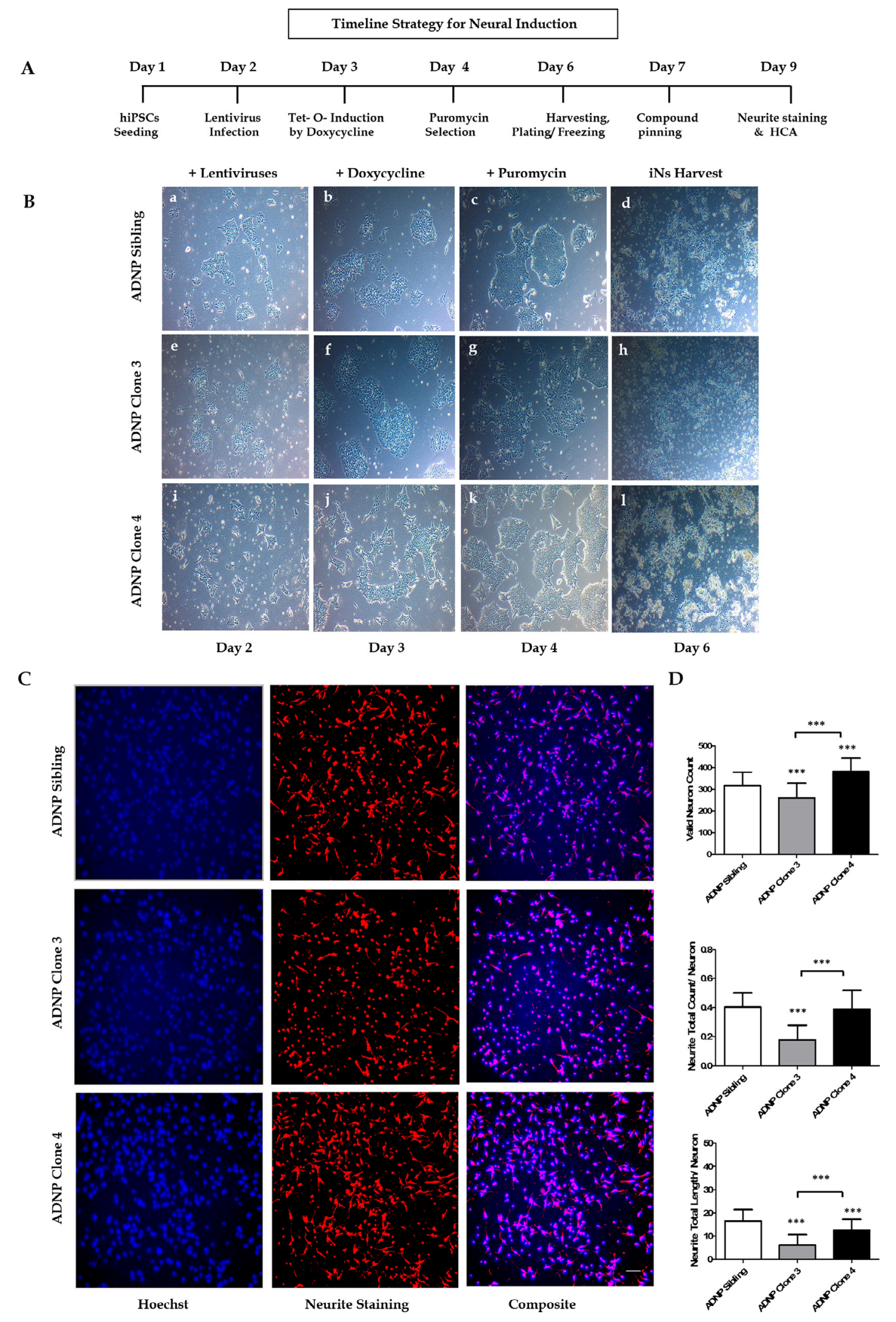

Fig 2. A) Time-line strategy used for neural induction and HCA, B) Representative phase contrast photomicrographs of hiPSCs (ADNP Sibling, and mutant clones 3 and 4) differentiating into neurons by Ngn2 neural induction system (4x magnification). C) Representative CellInsight fluorescent photomicrographs of iNs on Day 9 (5x objective), scalebar-100um D) Bar graphs represent comparison between study groups. Statistical analysis -One-way ANOVA followed by Tukeys' post hoc test; ${ }^{*} \mathrm{p}<0.05,{ }^{* *} \mathrm{p}<0.01,{ }^{* * *} \mathrm{p}<0.001, \mathrm{n}=400$ wells/ sample. Significance above each mutant is noted between parent and itself. Bracketed significance is noted between clones. 
A. ADNP Sibling
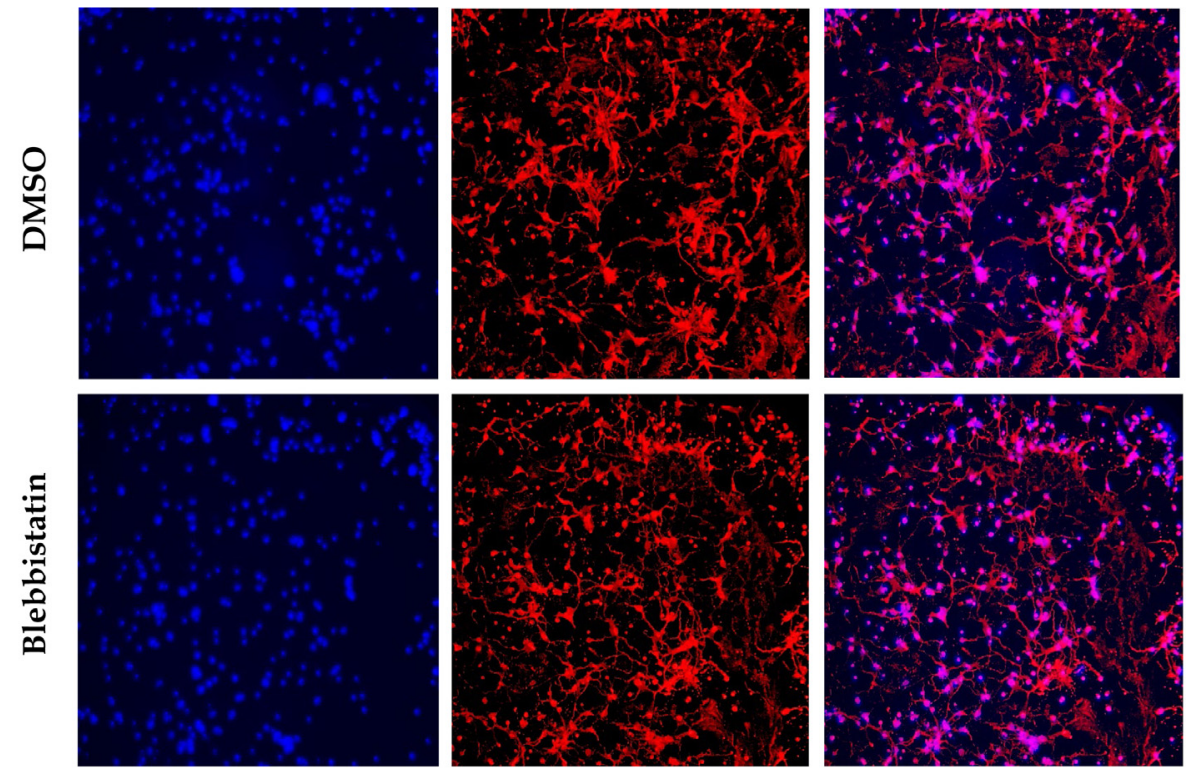

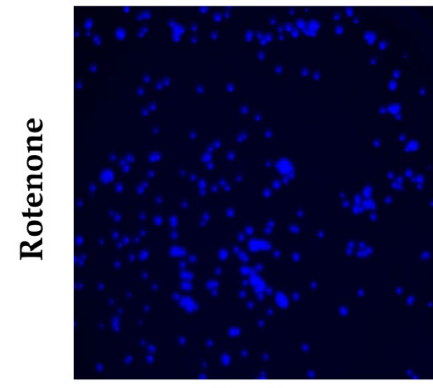

Hoechst

B. ADNP mutant- clone4
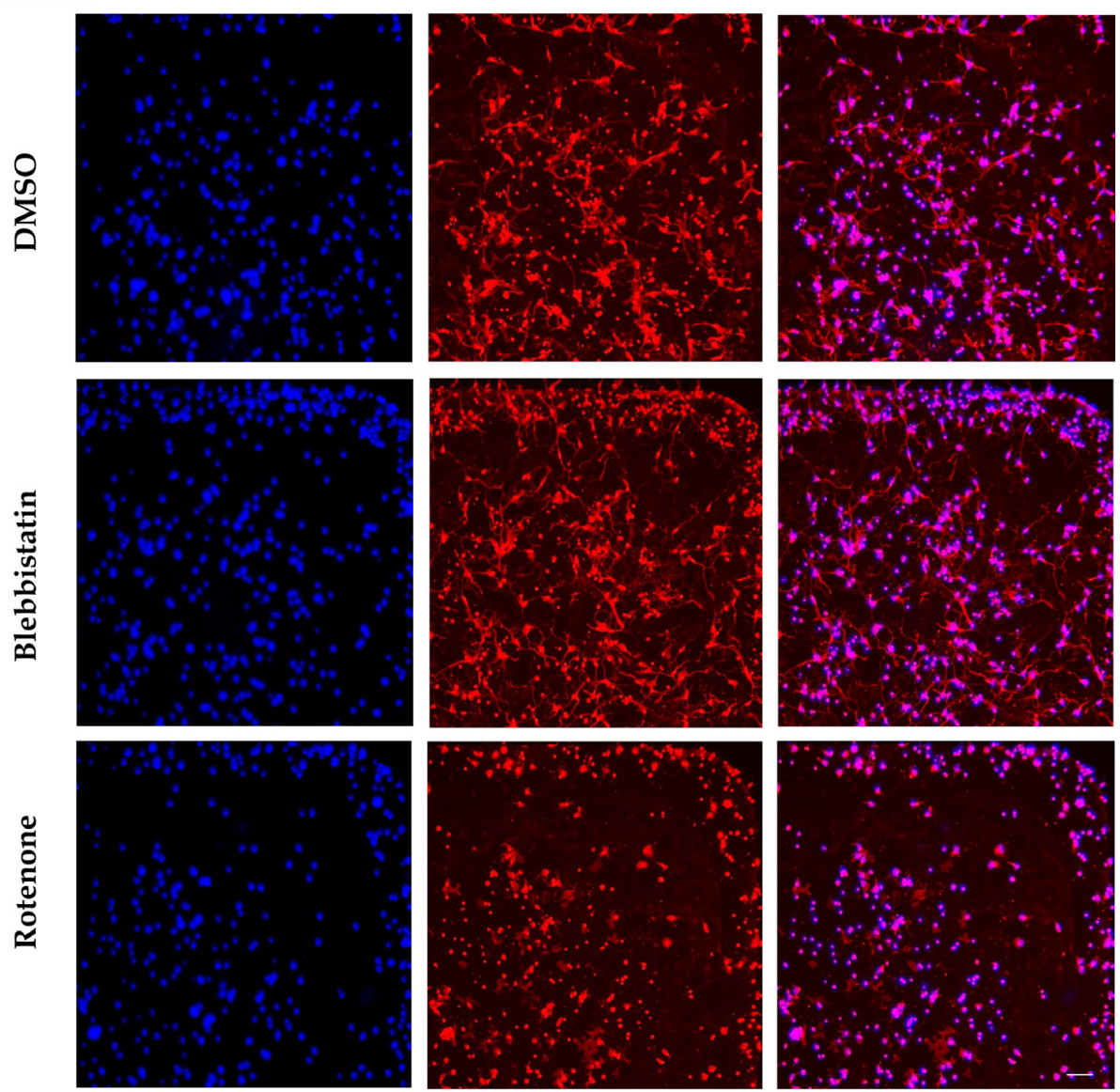

Neurite Staining

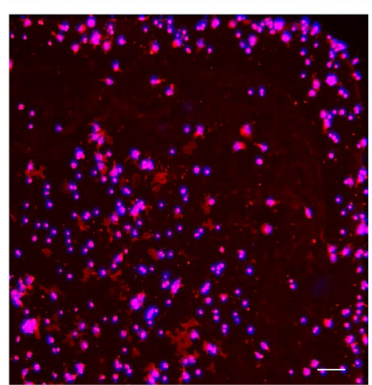

Composite

Fig 3. A and B) Representation fluorescent images of ADNP sibling and mutant 13 Clone 4 iNs treated with DMSO/ Blebbistatin or Rotenone, stained with Hoechst (Channel 1) and Neurite staining kit (Channel 2), 5x objective, CellInsight. 
$\mathbf{A}$

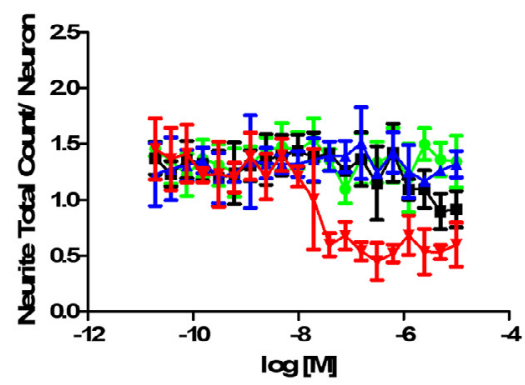

B

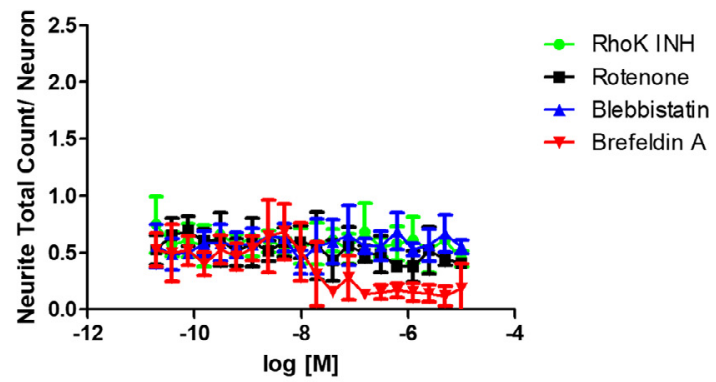

Neuroprotection

C



Activity- Raw Values
D

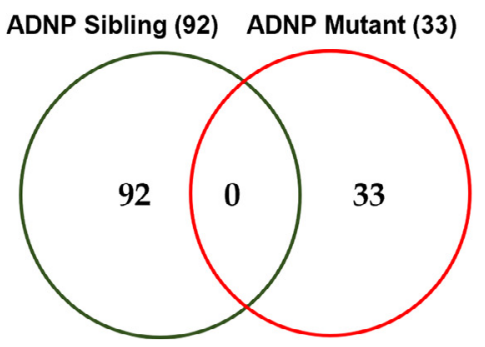

\section{Neurotoxicity}

E

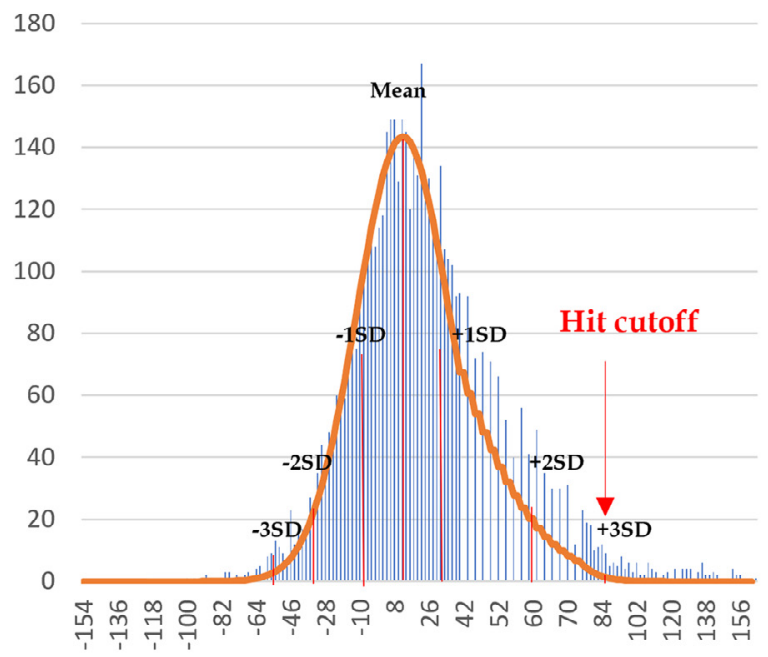

F

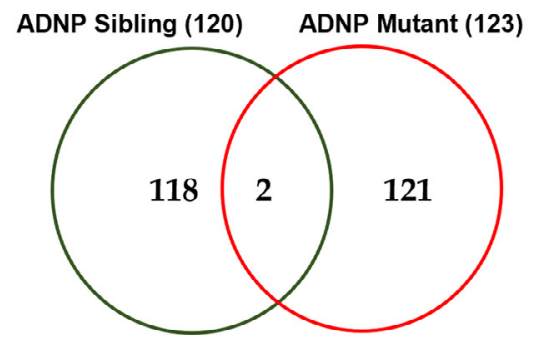

Average \% response

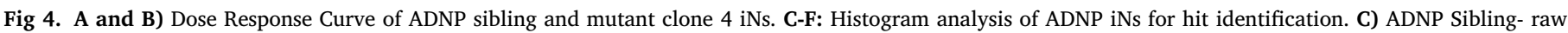

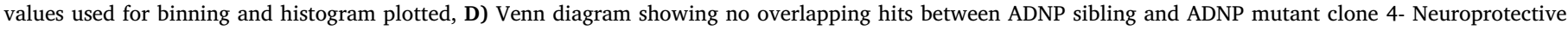

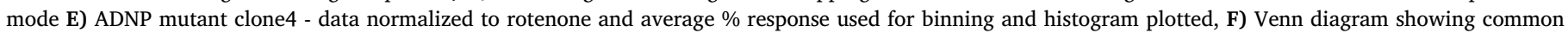
hits between ADNP sibling and ADNP mutant clone 4 - Neurotoxic mode. 


\section{A \\ Neuroprotective hits}

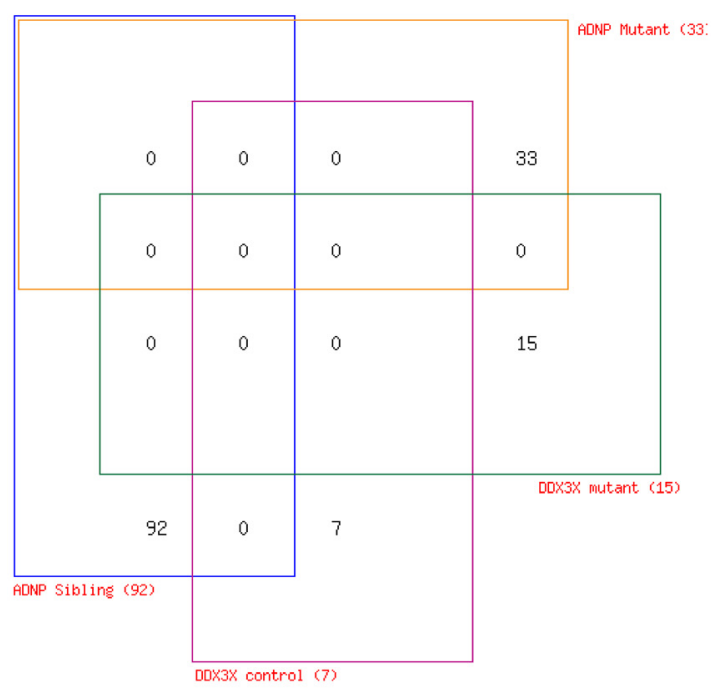

\section{Neurotoxic hits}

B



C

\section{Neurotoxic common hits}

\begin{tabular}{|l|r|}
\hline iNS & Hits \\
\hline ADNP sibling only & 117 \\
\hline ADNP mutant only & 50 \\
\hline DDX3X control only & 3 \\
\hline DDX3X mutant only & 31 \\
\hline ADNP sibling +ADNP mut & 1 \\
\hline ADNP sibling +DDX3X control & 0 \\
\hline ADNP mutant + DDX3X control & 2 \\
\hline ADNP sibling +DDX3X mutant & 0 \\
\hline ADNP mutant + DDX3X mutant & 16 \\
\hline DDX3X control + DDX3X mutant & 9 \\
\hline ADNP sibling +ADNP mutant + DDX3X control & 0 \\
\hline ADNP sibling +ADNP mutant + DDX3X mutant & 0 \\
\hline ADNP sibling +DDX3X control + DDX3X mutant & 1 \\
\hline ADNP mutant + DDX3X control + DDX3X mutant & 53 \\
\hline ADNP sibling + ADNP mutant + DDX3X control + DDX3X mutant & 1 \\
\hline
\end{tabular}

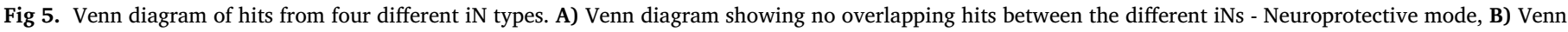
diagram showing overlapping hits between the different iNs - Neurotoxic mode, C) Venn diagram and table showing common hits in the neurotoxic mode.

experiments. Though of less interest, the neurotoxic hits were identified as those with the lowest standard deviation and highest level of activity in these iNs. The top 20 and 116 hits were cherry picked for testing ADNP and DDX3X iNs respectively. The compounds were tested in 3-fold dilution at 10 points in triplicate with the starting nominal concentration of $15 \mathrm{uM}$. Sample data for neurotoxicity and neuroprotectivity in ADNP sibling and mutant are represented in Fig. 6 and Sup. Fig. 9. Following the dose response experiment, Scripps and Icahn School of Medicine teams selected the compounds showing best neuroprotectivity. This was based on four separate and independent investigators selecting molecules based on their own interpretation of the concentration response visualization across all concentrations for all iNs. Venn analysis was done on all their choices and 19 common compounds were identified. Among the 19 hits, 3 were found to be overlapping between all the four different in types tested. Thirteen common neurotoxic hits were identified between the four different iNs (Sup. Fig. 10A \& B). Dose response data on the toxic effect of one of the neurotoxic hits tested on HEK and iNs are shown in Sup. Fig. 10C. Three neuroprotective and one neurotoxic compound were selected out of these common hits for downstream functional analysis.

\section{Discussion}

Inadequacy of animal models and failure of clinical trials drive the demand for deriving humanized models of ASD to understand and correct the disease pathology. Few studies are available to date that used patient derived cell-based assay for ASD. To address this, we set out to establish and develop assays for ASD using human relevant models for HTS.

In this study we attempted to differentiate many hiPSC cell lines carrying different ASD mutations into neurons. We observed differences in the growth and yield of these cell lines. Among the CRISPRed hiPSCs used SHANK3 Clone1 and Clone 10 were rapid in growth compared to the parent and other mutants. Different dissociation enzymes for hiPSCs, different passaging and neural induction time points were optimized for each cell line. FOXP1 and SHANK3 mutant hiPSCs showed spontaneous 


\section{Compound ID Structure}
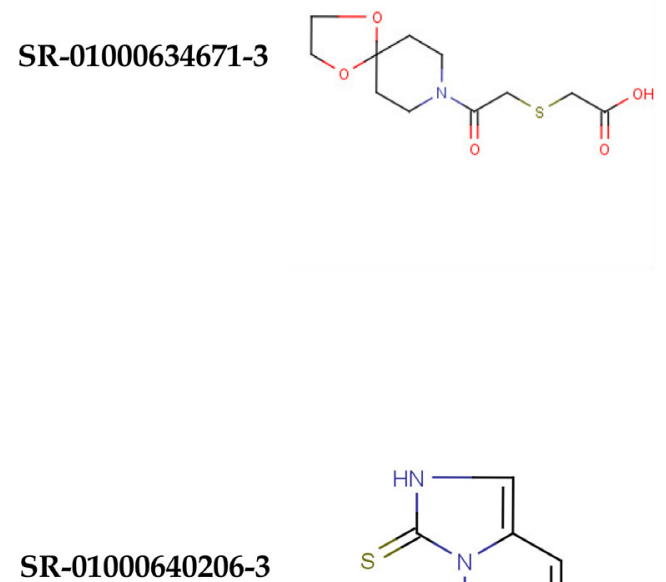<smiles>S=c1[nH]cc2ccccn12</smiles>

SR-01000645144-3

\section{Dose Response}


Fig 6. A sample data file showing the dose response of ADNP mutant clone 4 iNs to the neuroprotective Compounds.

differentiation resembling the neural rosettes or neurons. Hence, hiPSCs at lower passage number were used for neural induction assays. Further, patient derived hiPSCs had faster growth rate compared to the CRISPRed hiPSCs. However, all the hiPSCs cell lines were able to differentiate into glutamatergic neurons irrespective of the mutations suggesting the mutations did not compromise neurogenesis. iNs derived from these hiPSCs were grown on 384 plates were compared for phenotypic differences. The other challenges encountered, and the improvisations performed are listed in Table 1 . These optimized conditions allowed us to prepare ample quantities of hiPSCs for neuronal derivation and millions of neurons for HCA in a couple of weeks with lesser cost.

To recapitulate the human disease phenotype, we used hiPSC derived from ASD patients with ADNP mutant and compared the results to an unaffected sibling. The assay miniaturized to 1536 format performed well and the results were comparable to 384 formats that we have published on recently. To validate its translational relevance, we screened the iNs against compounds that can restore the affected neuronal phenotype. The resulting hits were cherry picked and tested in concentration response experiments and common hits were identified between the different iNs tested.

Screening for enhancers of neurite count per neuron (i.e., outgrowth), while possible, is certainly more complicated than screening for compounds that diminish the same parameter. While we demonstrated HTS amenability, we also note that the controls for outgrowth are limiting and hence achieving robust Z's $>0.5$ was not possible. To alleviate this, we screened in triplicate to overcome any deficiencies and 
Table 1

Summary of optimization steps used for the assay protocol.

\begin{tabular}{|c|c|c|c|}
\hline S. No & Cell type & Challenges & Optimization \\
\hline 1 & $\begin{array}{l}\text { hiPSCs - Isogenic and ASD } \\
\text { variants }\end{array}$ & Slower growth rate and spontaneous differentiation & $\begin{array}{l}\text { a) Aggregate passaging performed by replacing TrypLE Select } \\
\text { with ReLeSR } \\
\text { b) Low passage cells were used for neural induction }\end{array}$ \\
\hline 2 & hiPSCs derived from patients & Faster growth rate & Passaged within $24-48 \mathrm{hrs}$ and split ratio increased \\
\hline 3 & $\begin{array}{l}\text { iNs derived from patient } \\
\text { hiPSCs }\end{array}$ & $\begin{array}{l}\text { Cells grew fast, got overcrowded, formed aggregates, and } \\
\text { detached from the flasks during neural induction procedure }\end{array}$ & $\begin{array}{l}\text { a) hiPSCs were dissociated with ReLeSR and seeding density } \\
\text { was calculated in a parallel flask dissociated with TrypLE } \\
\text { select } \\
\text { b) Lesser number of hiPSCs were seeded for neural induction } \\
\text { c) Neural induction was initiated the immediate next day of } \\
\text { seeding }\end{array}$ \\
\hline 4 & iNs & $\begin{array}{l}\text { Neuro-profiling algorithm: } \\
\text { 'Well features' used for analysis in which the nuclei count did } \\
\text { not correspond to the real number of neurons }\end{array}$ & $\begin{array}{l}\text { a) 'Cell features' was used that counts neurons per well } \\
\text { identified in Channel } 2 \\
\text { b) Data were normalized to per neuron basis }\end{array}$ \\
\hline 5 & iNs & $\begin{array}{l}\text { iNs were plated in } 384 \mathrm{wp} \text { and washed by hand pipetting. This } \\
\text { caused higher variation between the wells of the same sample } \\
\text { resulting in increased standard deviation }\end{array}$ & $\begin{array}{l}\text { a) iNs were seeded using FRD } \\
\text { b) Blue washer with gentle spin mode was used for washing } \\
\text { steps }\end{array}$ \\
\hline
\end{tabular}

hence created a robust HTS campaign in that way. To our knowledge this is the first study to develop and implement phenotypic assays to test thousands of drug like compounds on patient derived neurons. Thus, this study sets the stage for HTS on human neuronal models that can be used to test hundreds of thousands of pharmacologically active compounds on different ASD mutations ultimately aiming at finding treatments. In depth studies are underway to identify and demonstrate the functional aspects of the identified compounds on these neurons.

\section{Funding}

This work was supported by the Seaver Foundation, and it was done as part of the Scripps Research shared services HTS core.

\section{Declaration of Competing Interest}

The authors declare that they have no conflict of interest.

\section{Supplementary materials}

Supplementary material associated with this article can be found, in the online version, at doi:10.1016/j.slasd.2022.01.004.

\section{References}

[1] Lord C, Risi S, DiLavore PS, Shulman C, Thurm A, Pickles A. Autism from 2 to 9 years of age. Arch Gen Psychiatry 2006;63(6):694-701.

[2] Gaugler T, Klei L, Sanders SJ, Bodea CA, Goldberg AP, Lee AB, Mahajan M, Manaa D, Pawitan Y, Reichert J, Ripke S, Sandin S, Sklar P, Svantesson O, Reichenberg A, Hultman CM, Devlin B, Roeder K, Buxbaum JD. Most genetic risk for autism resides with common variation. Nat Genet 2014;46(8):881-5.

[3] Ronald A, Hoekstra RA. Autism spectrum disorders and autistic traits: a decade of new twin studies. Am J Med Genet B Neuropsychiatr Genet 2011;156b(3):255-74.

[4] Sandin S, Lichtenstein P, Kuja-Halkola R, Larsson H, Hultman CM, Reichenberg A. The familial risk of autism. Jama 2014;311(17):1770-7.

[5] Sullivan PF, Geschwind DH. Defining the genetic, genomic, cellular, and diagnostic architectures of psychiatric disorders. Cell 2019;177(1):162-83.

[6] Satterstrom FK, Kosmicki JA, Wang J, Breen MS, De Rubeis S, An JY, Peng M, Collins R, Grove J, Klei L, Stevens C, Reichert J, Mulhern MS, Artomov M, Gerges S, Sheppard B, Xu X, Bhaduri A, Norman U, Brand H, Schwartz G, Nguyen R, Guerrero EE, Dias C, Betancur C, Cook EH, Gallagher L, Gill M, Sutcliffe JS, Thurm A, Zwick ME, Børglum AD, State MW, Cicek AE, Talkowski ME, Cutler DJ, Devlin B, Sanders SJ, Roeder K, Daly MJ, Buxbaum JD. Large-scale exome sequencing study implicates both developmental and functional changes in the neurobiology of autism. Cell 2020;180(3):568-84 e23.

[7] Ergaz Z, Weinstein-Fudim L, Ornoy A. Genetic and non-genetic animal models for autism spectrum disorders (ASD). Reprod Toxicol 2016;64:116-40.
[8] Shinoda Y, Sadakata T, Furuichi T. Animal models of autism spectrum disorder (ASD): a synaptic-level approach to autistic-like behavior in mice. Exp Anim 2013;62(2):71-8

[9] Rakic P. Evolution of the neocortex: a perspective from developmental biology. Nat Rev Neurosci 2009;10(10):724-35.

[10] Sridharan B, Hubbs C, Llamosas N, Kilinc M, Singhera FU, Willems E, Piper DR, Scampavia L, Rumbaugh G, Spicer TP. A simple procedure for creating scalable phenotypic screening assays in human neurons. Sci Rep 2019;9(1):9000.

[11] Sumitha R, Manjunatha VM, Sabitha RK, Alladi PA, Nalini A, Rao LT, Chandrasekhar Sagar BK, Steinbusch HWM, Kramer BW, Sathyaprabha TN, Raju TR. Cerebrospinal fluid from patients with sporadic amyotrophic lateral sclerosis induces degeneration of motor neurons derived from human embryonic stem cells. Mol Neurobiol 2019;56(2):1014-34.

[12] Kathuria A, Nowosiad P, Jagasia R, Aigner S, Taylor RD, Andreae LC, Gatford NJF, Lucchesi W, Srivastava DP, Price J. Stem cell-derived neurons from autistic individuals with SHANK3 mutation show morphogenetic abnormalities during early development. Mol Psychiatry 2018;23(3):735-46.

[13] Lewis EMA, Meganathan K, Baldridge D, Gontarz P, Zhang B, Bonni A, Constantino JN, Kroll KL. Cellular and molecular characterization of multiplex autism in human induced pluripotent stem cell-derived neurons. Mol Autism 2019;10:51.

[14] Yi F, Danko T, Botelho SC, Patzke C, Pak C, Wernig M, Südhof TC. Autism-associated SHANK3 haploinsufficiency causes Ih channelopathy in human neurons. Science 2016;352(6286):aaf2669.

[15] Pintacuda G, Martín JM, Eggan KC. Mind the translational gap: using iPS cell models to bridge from genetic discoveries to perturbed pathways and therapeutic targets. Mol Autism 2021;12(1):10.

[16] Darville H, Poulet A, Rodet-Amsellem F, Chatrousse L, Pernelle J, Boissart C, Héron D, Nava C, Perrier A, Jarrige M, Cogé F, Millan MJ, Bourgeron T, Peschanski M, Delorme R, Benchoua A. Human pluripotent stem cell-derived cortical neurons for high throughput medication screening in autism: a proof of concept study in SHANK3 haploinsufficiency syndrome. EBioMedicine 2016;9:293-305.

[17] Zhang Y, Pak C, Han Y, Ahlenius H, Zhang Z, Chanda S, Marro S, Patzke C, Acuna C, Covy J, Xu W, Yang N, Danko T, Chen L, Wernig M, Südhof TC. Rapid single-step induction of functional neurons from human pluripotent stem cells. Neuron 2013;78(5):785-98.

[18] Jang Y, Choi J, Park N, Kang J, Kim M, Kim Y, Ju JH. Development of immunocompatible pluripotent stem cells via CRISPR-based human leukocyte antigen engineering. Exp Mol Med 2019;51(1):1-11.

[19] Breen MS, Browne A, Hoffman GE, Stathopoulos S, Brennand K, Buxbaum JD, Drapeau E. Transcriptional signatures of participant-derived neural progenitor cells and neurons implicate altered Wnt signaling in Phelan-McDermid syndrome and autism. Molecular Autism 2020;11(1):53.

[20] Sridharan B, Hubbs C, Llamosas N, Kilinc M, Singhera FU, Willems E, Piper DR, Scampavia L, Rumbaugh G, Spicer TP. A Simple Procedure for Creating Scalable Phenotypic Screening Assays in Human Neurons. Scientific Reports 2019;9(1):9000.

[21] Baillargeon P, Fernandez-Vega V, Sridharan BP, Brown S, Griffin PR, Rosen H, Cravatt B, Scampavia L, Spicer TP. The scripps molecular screening center and translational research institute. SLAS Discov 2019;24(3):386-97.

[22] Smith E, Davis-Gardner ME, Garcia-Ordonez RD, Nguyen TT, Hull M, Chen E, Baillargeon P, Scampavia L, Strutzenberg T, Griffin PR, Farzan M, Spicer TP. High-throughput screening for drugs that inhibit papain-like protease in SARS-CoV-2. SLAS Discov 2020;25(10):1152-61.

[23] R Studio | Open source \& professional software for data science teams. https://www.rstudio.com/ (accessed September 2021).

[24] R: The R Project for Statistical Computing https://www.r-project.org/ (accessed September 2021). 
[25] Create Elegant Data Visualizations Using the Grammar of Graphics - ggplot2. https://ggplot2.tidyverse.org/ (accessed September 2021).

[26] Hacohen-Kleiman G, Sragovich S, Karmon G, Gao AYL, Grigg I, Pasmanik-Chor M, Le A, Korenková V, McKinney RA, Gozes I. Activity-dependent neuroprotective protein deficiency models synaptic and developmental phenotypes of autism-like syndrome. J Clin Invest 2018;128(11):4956-69.
[27] Pearson CA, Moore DM, Tucker HO, Dekker JD, Hu H, Miquelajáuregui A, Novitch BG. Foxp1 regulates neural stem cell self-renewal and bias toward deep layer cortical fates. Cell Rep 2020;30(6):1964-81 .e3.

[28] Kathuria A, Nowosiad P, Jagasia R, Aigner S, Taylor RD, Andreae LC, Gatford NJF, Lucchesi W, Srivastava DP, Price J. Stem cell-derived neurons from autistic individuals with SHANK3 mutation show morphogenetic abnormalities during early development. Molecular Psychiatry 2018;23(3):735-46. 